\title{
Effect of Thermo- Diffusion and Dissipation on Double Diffusive Heat and Mass Transfer Flow Of A Kuvshinski Fluid
}

\author{
V.Suresh Babu ${ }^{\# 1}$ K. Rama Krishna Reddy ${ }^{\# 2}$ \\ ${ }^{I}$ Assistant Professor in Mathematics(H\&S), Ravindra college of Engg. for women, Kurnool, A.P., India \\ ${ }^{2}$ Professor in Dpartment of Mathematics, Raghavendra Institute of Pharamaceutical Education and Research, \\ Anantapuram, A.P. ,India
}

\begin{abstract}
In this paperthe combined influence of chemical reaction, thermo diffusion and dissipation on convective heat and mass transfer flow of a Kuvshinski fluid past a vertical plate embedded in a porous medium is discussed. The equations governing the flow, heat and mass transfer have been solved by a regular perturbation technique. The effect of chemical reaction parameter $(K)$, thermo-diffusion parameter $\left(S_{r}\right)$, Eckert number $\left(E_{c}\right)$, heat source parameter $(\alpha)$ and time $(t)$ on the velocity, temperature and concentration distributions are discussed. It is observed that the temperature and concentration reduces with increase in the strength of the heat source parameter where as the velocity depreciates with increase in heat source parameter. In the presence of $E_{c}$, it is observe that higher the dissipative heat smaller the velocity, temperature, and concentration in the flow field and we notice an enhancement in the case of time ( $t)$.
\end{abstract}

Keywords: Kuvshinski fluid, MHD, Thermo-Diffusion, Chemical Reaction

\section{Introduction}

The convection problem in a porous medium has important applications in geothermal reservoirs and geothermal extractions. The process of heat and mass transfer is encountered in aeronautics, fluid fuel nuclear reactor, chemical process industries and many engineering applications in which the fluid is the working medium. The unsteady fluid flow past a moving plate in the presence of free convection and radiation were studies by Monsure [8], Raptis and Perdikis [12], Ganesan and Loganathan [3], Mbeledogu et al. [9], Makinde [7], and Abdus -Satter and Hamid Kalim [1],Cookeyet al.[2],Hassanien.et al.[5],Kim [6] ,Raptis, etal. $[13,14,15]$. All these studies have been confined to unsteady flow in a non-porous medium.

The study of heat generation or absorption effects in moving fluids is important in view of several physical problems, such as fluids undergoing exothermic or endothermic chemical reactions. Combined heat and mass transfer problems with chemical reaction are of importance in many processes and have, therefore received a considerable amount of attention in recent years. For example in the power industry among the methods of generation electric power is one in which electrical energy is extracted directly form a moving conducting fluids. Many practical diffusive operations involve the molecular diffusion of a species in the presence of chemical reaction within or at the boundary. There are two types of reactions; a homogeneous reaction is one that occurs uniformly throughout a give phase. The species generation in a homogeneous reaction is analogous to internal source of heat generation. In constraint a heterogeneous reaction takes place in a restricted region or within the boundary of a phase. It can therefore be treated as a boundary condition similar to the constant heat flux condition in heat transfer the study of heat and mass transfer withchemical reaction is of great practical importance to engineers and scientists because of its almost universal occurrence in many branches of science and engineering. Muthucumaraswamy and Ganesan [11] studied first-order chemical reaction on flow past on impulsively started vertical plate with uniform heat and mass flux. Recently, Mohamed [10] has discussed double-diffusive convection-radiation interaction on unsteady MHD flow over a vertical moving porous plate with heat generation and Soret effects. Recently Kuvshinski fluid is attracting the attention of many researchers [16-18]. Ravikumar et al. [19] addressedMHD three dimensional Couette flow past a porous plate with heat transfer. Numerical study of Magneto-Convective and radiation absorption fluid flow past an exponentially accelerated vertical porous plate with variable temperature and concentration in the presence of Soret and Dufour effects was reported by Umamaheswar et al. [20]. MHD transient free convection and chemically reactive flow past a porous vertical plate with radiation and temperature gradient dependent heat source in slip flow regime was considered by Rao et al.[21]. Important applications of double diffusive flows are highlighted by few authors [22-27]. Influence of thermal diffusion on convective flows was also addressed by several authors [28-30].

In spite of all these studies the effect of Kuvshinski fluid on unsteady MHD double diffusive free convection for a heat generating fluids with thermal radiation and chemicals reaction has received more 
attention. Hence in this paper combined influence of chemical reaction, thermo diffusion and dissipation on convective heat and mass transfer flow of a Kuvshinski fluid past a vertical plate embedded in a porous medium is investigated.

\section{Formulation Of The Problem}

We consider a rectangular Cartesian system $\mathrm{O}(\mathrm{x}, \mathrm{y})$ with $\mathrm{x}$-axis along the plate and $\mathrm{y}$-axis normal to it. Initially it is assumed that the plate and the fluid are at the same temperature $T_{\infty}$ and the concentration level $C_{\infty}$ everywhere in the fluid. The radiating heat flux in the x-direction is considered negligible in comparison to that in the y-direction. The fluid assumed to be gray emitting and absorbing radiation but non-scattering medium. All the fluid properties are considered constant except in the influence of the density variation with temperature in the body-force term. Under the above assumptions the equations governing the flow, heat and mass transfer are

Continuity equation is

$$
\frac{\partial v}{\partial y}=0
$$

Momentum equation is

$$
\left(1+\lambda \frac{\partial}{\partial t}\right) \frac{\partial u}{\partial t}+v \frac{\partial u}{\partial y}=\beta g\left(T-T_{\infty}\right)+\beta^{\bullet} g\left(C-C_{\infty}\right)+v \frac{\partial^{2} u}{\partial y^{2}}-\frac{v}{k}\left(1+\lambda \frac{\partial}{\partial t}\right) u-\frac{\sigma \mu_{e}^{2} H_{o}^{2}}{\rho} u
$$

Energy equation

$$
\left(1+\lambda \frac{\partial}{\partial t}\right) \frac{\partial T}{\partial t}+v \frac{\partial T}{\partial y}=\frac{k_{0}}{\rho C_{p}} \frac{\partial^{2} T}{\partial y^{2}}+\frac{v}{C_{p}}\left(\frac{\partial u}{\partial y}\right)^{2}+Q\left(T-T_{\infty}\right)
$$

Diffusion equation

$$
\left(1+\lambda \frac{\partial}{\partial t}\right) \frac{\partial C}{\partial t}+v \frac{\partial C}{\partial y}=D_{1} \frac{\partial^{2} C}{\partial y^{2}}-k_{r}^{\prime}\left(C-C_{\infty}\right)+k_{11} \frac{\partial^{2} T}{\partial y^{2}}
$$

The initial and boundary conditions are

$$
\begin{aligned}
& u=0, T=T_{w}, C=C_{w} \quad \text { at } y=0 \\
& u \rightarrow 0, T \rightarrow T_{\infty}, C \rightarrow C_{\infty} \text { as } y \rightarrow \infty
\end{aligned}
$$

Where $\mathrm{u}$ and $\mathrm{v}$ are the parallel and perpendicular components of velocity to the plate, $\mathrm{t}$ is the time, $\rho$ is the density, $v$ is the kinematic viscosity, $\mathrm{Cp}$ is the specific heat at constant pressure, $\mathrm{g}$ is the acceleration due to gravity, $\beta$ is the volumetric coefficient of thermal expansion, $\beta^{\bullet}$ is the volumetric coefficient of concentration expansion. T, $\mathrm{C}$ are the temperature and Species concentration in the fluid region. $\mathrm{T}_{\mathrm{w}}, \mathrm{C}_{\mathrm{w}}$ are wall temperature and Concentration, $T_{\infty} \& C_{\infty}$ are the free stream temperature and concentration in fluid far away from the plate, $\mathrm{k}_{\mathrm{o}}$ is the thermal conduction, $\mathrm{D}_{1}$ is the mass diffusivity, $\mathrm{q}_{\mathrm{r}}$ is the radiation heat flux, $\mathrm{k}$ is the permeability of the porous medium, $k_{r}^{\prime}$ is the chemical reaction coefficient, $\mathrm{Q}$ is the strength of the heat source, $\mathrm{k}_{11}$ is the cross diffusivity. The third term on the right hand side of equation (2.3) represents radiative heat flux term.

The equation of continuity (1) gives

$$
v=-v_{0}
$$

Where $\mathrm{v}_{\mathrm{o}}$ is the constant suction velocity normal to the plate.

In view of (6) the equations (2), (3) and (4) now reduce to

$$
\begin{aligned}
& \left(1+\lambda \frac{\partial}{\partial t}\right) \frac{\partial u}{\partial t}-v_{0} \frac{\partial u}{\partial y}=\beta g\left(T-T_{\infty}\right)+\beta^{\bullet} g\left(C-C_{\infty}\right)+v \frac{\partial^{2} u}{\partial y^{2}}-\frac{v}{k}\left(1+\lambda \frac{\partial}{\partial t}\right) u-\frac{\sigma \mu_{e}^{2} H_{o}^{2}}{\rho} u \\
& \left(1+\lambda \frac{\partial}{\partial t}\right) \frac{\partial T}{\partial t}-v_{0} \frac{\partial T}{\partial y}=\frac{k_{0}}{\rho C_{p}} \frac{\partial^{2} T}{\partial y^{2}}+\frac{v}{C_{p}}\left(\frac{\partial u}{\partial y}\right)^{2}+Q\left(T-T_{\infty}\right)
\end{aligned}
$$




$$
\left(1+\lambda \frac{\partial}{\partial t}\right) \frac{\partial C}{\partial t}-v_{0} \frac{\partial C}{\partial y}=D_{1} \frac{\partial^{2} C}{\partial y^{2}}-k_{r}^{\prime}\left(C-C_{\infty}\right)+k_{11} \frac{\partial^{2} T}{\partial y^{2}}
$$

On introducing the following non-dimensional variables

$$
u^{\prime}=\frac{u}{v_{0}}, y^{\prime}=\frac{y v_{0}}{v}, \theta=\frac{T-T_{\infty}}{T_{w}-T_{\infty}}, \phi=\frac{C-C_{\infty}}{C_{w}-C_{\infty}}, t^{\prime}=\frac{v_{o}^{2} t}{v},
$$

The governing equations reduce to

$$
\begin{aligned}
& \alpha_{1} \frac{\partial u}{\partial t}+\alpha_{2} \frac{\partial^{2} u}{\partial t^{2}}=G(\theta+N \phi)+\frac{\partial^{2} u}{\partial y^{2}}+\frac{\partial u}{\partial y}-\left(M^{2}+D^{-1}\right) u \\
& \operatorname{Pr} \frac{\partial \theta}{\partial t}+\operatorname{Pr} \alpha_{2} \frac{\partial^{2} \theta}{\partial y^{2}}+\frac{\partial^{2} \theta}{\partial y^{2}}+\operatorname{Pr} \frac{\partial \theta}{\partial y}+\operatorname{Pr} E c\left(\frac{\partial u}{\partial y}\right)^{2}-\alpha \operatorname{Pr} \theta \\
& S c \frac{\partial \phi}{\partial t}+S c \alpha_{2} \frac{\partial^{2} \phi}{\partial t^{2}}=\frac{\partial^{2} \phi}{\partial y^{2}}+S c \frac{\partial \phi}{\partial y}-\left(k_{r} S c\right) \phi+\frac{S c S o}{N} \frac{\partial^{2} \theta}{\partial y^{2}}
\end{aligned}
$$

Where

$$
\begin{aligned}
& M^{2}=\frac{\sigma \mu_{e}^{2} H_{o}^{2} v}{\rho v_{o}^{2}} \text { (Magnetic parameter), } D^{-1}=\frac{v^{2}}{k v_{o}^{2}} \quad \text { (Darcy parameter) } \\
& G=\frac{\beta g\left(T_{w}-T_{\infty}\right) v}{v_{o}^{3}} \text { (Grashof number), } \operatorname{Pr}=\frac{\rho C_{p}}{k_{o}} \text { (Prandtl Number) }
\end{aligned}
$$

$N_{1}=\frac{\beta_{R} k_{0}}{4 \sigma^{\bullet} T_{\infty}^{3}}$, (Radiation parameter), $N=\frac{\beta^{\bullet}\left(C_{w}-C_{\infty}\right)}{\beta\left(T_{w}-T_{\infty}\right)}$ (Buoyancy ratio)

$k r=\frac{k_{r}^{\prime} v}{v_{o}^{2}}$ (Chemical reaction parameter) $\alpha=\frac{Q v}{v_{o}^{2}}$ (Heat source parameter)

$S r=\frac{k_{11}\left(T_{w}-T_{\infty}\right)}{\left(C_{w}-C_{\infty}\right)} \quad$ (Soret parameter), $\alpha_{1}=1+\frac{v \lambda}{k}, \alpha_{2}=\frac{\lambda v_{o}^{2}}{v}$

The corresponding boundary conditions are

$$
\begin{aligned}
& u=0, \theta=1, \phi=1 \text { at } y=0 \\
& u \rightarrow 0, \theta \rightarrow 0, \phi \rightarrow 0 \text { as } y \rightarrow \infty
\end{aligned}
$$

\section{Method Of Solution}

To solve the coupled non-linear equations (11) - (13) subject to the boundary conditions (14), we may represent the velocity $(\mathrm{u})$, temperature $(\theta)$, concentration $(\phi)$ of the fluid in powers of Eckert number $(\mathrm{Ec})$ assuming it to be very small. Hence we can write

$$
\begin{aligned}
& u=u_{0}+E c u_{1} e^{-n t} \\
& \theta=\theta_{0}+E c \theta_{1} e^{-n t} \\
& \phi=\phi_{0}+E c \phi_{1} e^{-n t}
\end{aligned}
$$

Substituting (15) into the equations (11) - (13) and equating harmonic, non-harmonic terms and neglecting $\mathrm{Ec}^{2}$ and higher order terms, we get

$$
\begin{aligned}
& \frac{d^{2} u_{0}}{d y^{2}}+\frac{d u_{0}}{d y}-\left(M^{2}+D^{-1}\right) u_{0}=-G\left(\theta_{0}+N \phi_{0}\right) \\
& \frac{d^{2} u_{1}}{d y^{2}}+\frac{d u_{1}}{d y}-\left(M^{2}+D^{-1}-n \alpha_{1}+n^{2} \alpha_{2}\right) u_{1}=-G\left(\theta_{1}+N \phi_{1}\right)
\end{aligned}
$$




$$
\begin{aligned}
& \frac{d^{2} \theta_{0}}{d y^{2}}+\operatorname{Pr} \frac{d \theta_{0}}{d y}-\alpha \operatorname{Pr} u_{0}=0 \\
& \frac{d^{2} \theta_{1}}{d y^{2}}+\operatorname{Pr} \frac{d \theta_{1}}{d y}+\left(n \operatorname{Pr}-n^{2} \operatorname{Pr} \alpha_{2}-\alpha \operatorname{Pr}\right) \theta_{1}=-\operatorname{Pr}\left(\frac{d u_{0}}{d y}\right)^{2} \\
& \frac{d^{2} \phi_{0}}{d y^{2}}+S c \frac{d \phi_{0}}{d y}-\left(S c k_{r}\right) \phi_{0}=-S c S r \frac{d^{2} \theta_{0}}{d y^{2}} \\
& \frac{d^{2} \phi_{1}}{d y^{2}}+S c \frac{d \phi_{1}}{d y}+\left(n S c-n^{2} S c \alpha_{2}-K r S c\right) \phi_{1}=-S c S r \frac{d^{2} \theta_{1}}{d y^{2}}
\end{aligned}
$$

The corresponding boundary conditions now become

$$
\begin{aligned}
& u_{0}=0, u_{1}=0, \theta_{0}=1, \theta_{1}=0, \phi_{0}=1, \phi_{1}=0 \text { at } y=0 \\
& u_{0} \rightarrow 0, u_{1} \rightarrow 0, \theta_{0} \rightarrow 0, \theta_{1} \rightarrow 0, \phi_{0} \rightarrow 0, \phi_{1} \rightarrow 0 \text { as } y \rightarrow \infty
\end{aligned}
$$

Solving the equations (16) - (21) under the boundary conditions (22) we get

$$
\begin{aligned}
& \theta_{o}=\exp \left(-m_{1} y\right) \\
& \phi_{0}=\exp \left(-m_{2} y\right)+a_{2}\left(\exp \left(-m_{1} y-\exp \left(-m_{2} y\right)\right)\right. \\
& u_{0}=a_{3}\left(\exp \left(-m_{1} y\right)-\exp \left(-m_{3} y\right)\right)+a_{4}\left(\exp \left(-m_{2} y\right)-\exp \left(-m_{3} y\right)\right) \\
& \theta_{1}=a_{16} \exp \left(-m_{4} y\right)+f_{2}(y) \\
& f_{2}(y)=a_{6} \exp \left(-2 m_{1} y\right)+\left(a_{7}+a_{10}+a_{13}\right) \exp \left(-2 m_{3} y\right)-\left(a_{8}+a_{14}\right) \exp \left(-\left(m_{1}+m_{3}\right) y\right)+ \\
&+a_{9} \exp \left(-2 m_{2} y\right)-a_{11} \exp \left(-\left(m_{2}+m_{3}\right) y+a_{12} \exp \left(-\left(m_{1}+m_{2}\right) y\right)\right. \\
& \phi_{1}=a_{26} \exp \left(-m_{5} y\right)+f_{3}(y) \\
& f_{3}(y)=a_{16} \exp \left(-m_{4} y\right)+a_{17} \exp \left(-2 m_{1} y\right)+\left(a_{18}+a_{21}\right) \exp \left(-2 m_{3} y\right)+ \\
&+\left(a_{19}+a_{24}\right) \exp \left(-\left(m_{1}+m_{3}\right) y\right)+a_{20} \exp \left(-2 m_{2} y\right)+\left(a_{22}+a_{25}\right) \exp \left(-\left(m_{2}+m_{3}\right) y\right) \\
&+a_{23} \exp \left(-\left(m_{1}+m_{2}\right) y\right) \\
& u_{1}=a_{35} \exp \left(-m_{6} y\right)+f_{4}(y) \\
& f_{4}(y)=a_{27} \exp \left(-m_{4} y\right)+a_{28} \exp \left(-2 m_{1} y\right)+a_{29} \exp \left(-2 m_{3} y\right)+ \\
&+\left(a_{30}+a_{34}\right) \exp \left(-\left(m_{1}+m_{3}\right) y\right)+a_{31} \exp \left(-2 m_{2} y\right)+ \\
& \quad+a_{32} \exp \left(-\left(m_{2}+m_{3}\right) y\right)+a_{33} \exp \left(-\left(m_{1}+m_{2}\right) y\right)
\end{aligned}
$$

\section{Skin Friction, Nusselt Number And Sherwood Number}

The skin friction $(\tau)$ at the wall $\mathrm{y}=0$ is given by

$$
\tau=\left(\frac{\partial u}{\partial y}\right)_{y=0}=a_{36}+E c a_{37} e^{-n t}
$$

The rate of heat transfer (Nusselt Number) at the wall $\mathrm{y}=0$ is given by

$$
N u=\left(\frac{\partial \theta}{\partial y}\right)_{y=0}=-m_{4}+E c a_{38} e^{-n t}
$$

The rate of mass transfer (Sherwood Number) at the wall $\mathrm{y}=0$ is given by

$$
S h=\left(\frac{\partial \phi}{\partial y}\right)_{y=0}=a_{39}+E c a_{40} e^{-n t}
$$

Particular case: In the absence of thermo-diffusion and heat sources the result are in good agreement with the Giresh Kumar etal. [4]. 


\section{Results And Discussion}

In this analysis we investigate the effect of chemical reaction and thermo-diffusion on MHD Convective heat and mass transfer flow of a Kuvshinski fluid past a vertical plate embedded in a saturated porous medium.

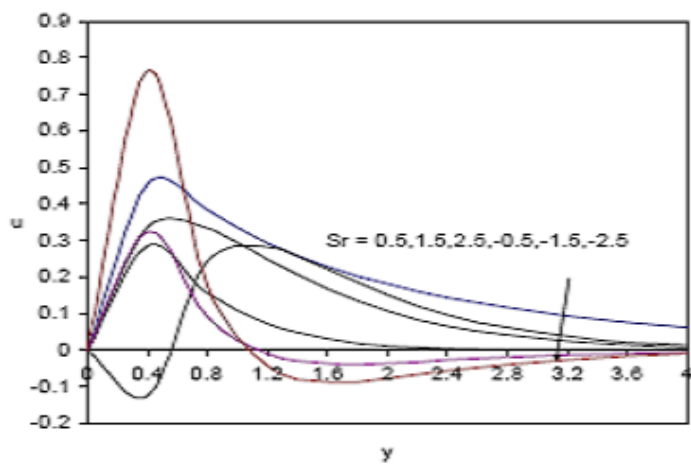

Fig. 1 : Variation of $\mathrm{u}$ with $\mathrm{S}_{\text {, }}$ $\mathrm{G}=10^{3}, \mathrm{M}=2, \mathrm{D}^{-1}=2, \mathrm{~N}=1, \mathrm{~S} c=1.3, \mathrm{~K}=0.5$,

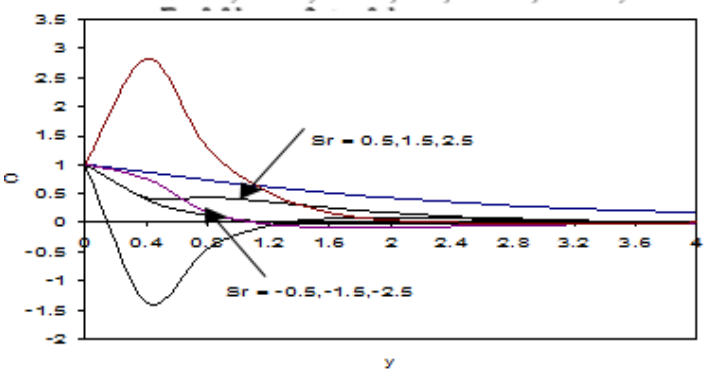

$\mathrm{G}=10^{2} \begin{aligned} & \text { Fig. } 3: \text { V ariation of } \mathrm{C} \text { with } \mathrm{S}_{\mathrm{r}} \\ & \mathrm{M}=2, \mathrm{D}^{-1}=2, \mathrm{~N}=1 ., \mathrm{S}=1.3 . \mathrm{K}=0.5 . \\ & \mathrm{F}_{\mathrm{s}}=0.01, \alpha=2, \mathrm{t}=0.1\end{aligned}$

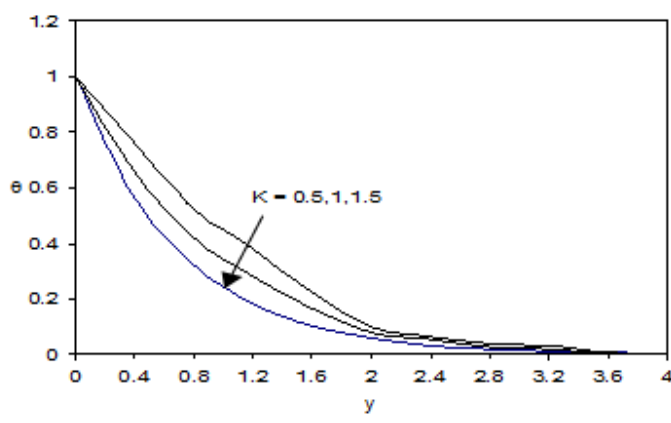

Fig. 5 : $V$ ariation of $\theta$ with $K$

$\mathrm{G}=10^{2}, \mathrm{M}=2, \mathrm{D}^{-1}=2, \mathrm{~N}=1, \mathrm{~S}_{\mathrm{s}}=1.3, \mathrm{~S}_{\mathrm{r}}=0.5$ $E_{s}=0.01, \alpha=2, t=0.1$

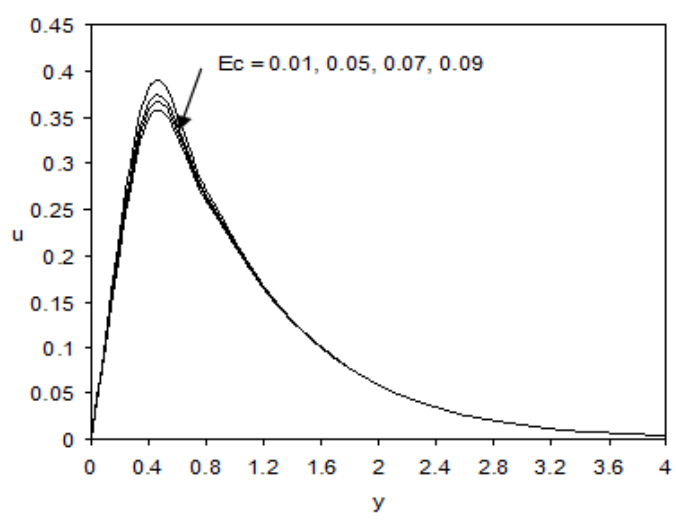

Fig. 7: Variation of $\mathrm{u}$ with $\mathrm{Ec}$

$\mathrm{G}=10^{3}, \mathrm{M}=2, \mathrm{D}^{-1}=2, \mathrm{~N}=1, \mathrm{Sc}=1.3, \mathrm{~S}_{\mathrm{f}}=0.5$

$\mathrm{K}=0.5, \alpha=2, \mathrm{t}=0.1$

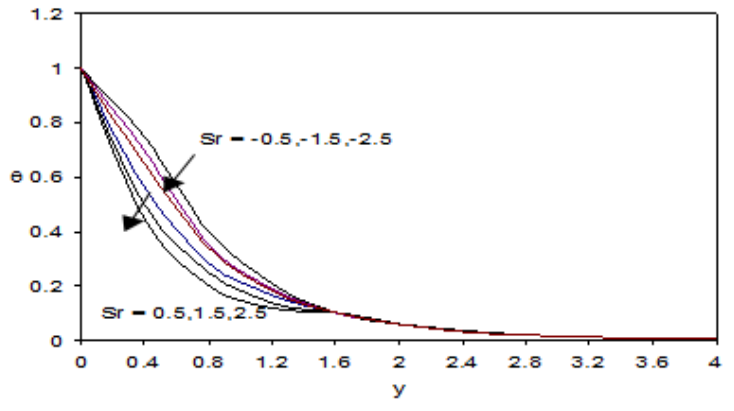

Fig. 2 : Variation of $\theta$ with $S_{r}$

$\mathrm{S}_{\mathrm{s}}=1.3, \mathrm{~K}=0.5$

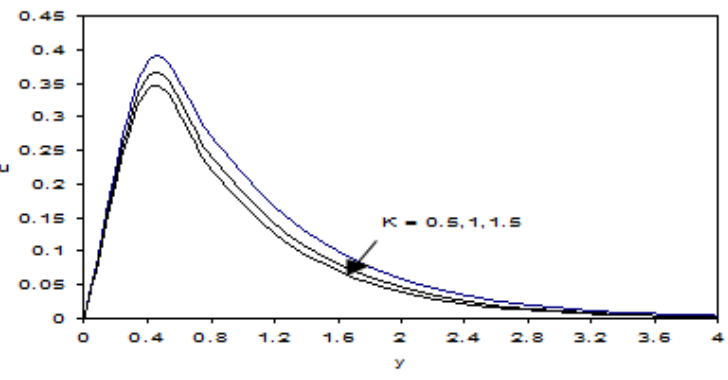

Fig.4: Variation of u with $\mathrm{K}$
$\mathrm{G}=1 \mathrm{O}^{2} . \mathrm{M}=2, \mathrm{D}^{-1}=2, \mathrm{~N}=1 . \mathrm{S}_{c}=1.3 . \mathrm{S}_{\mathrm{x}}=0.5$

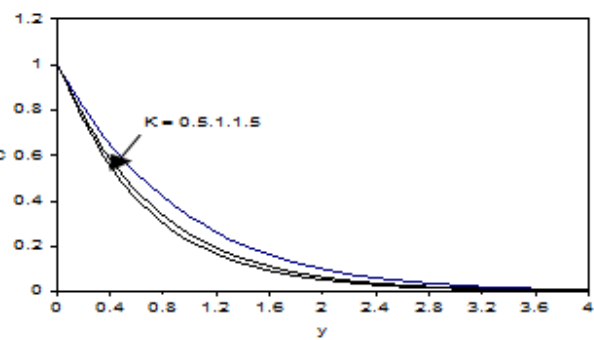

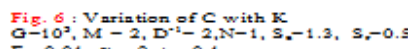
E. $-0.01, \alpha-2, t-0.1$

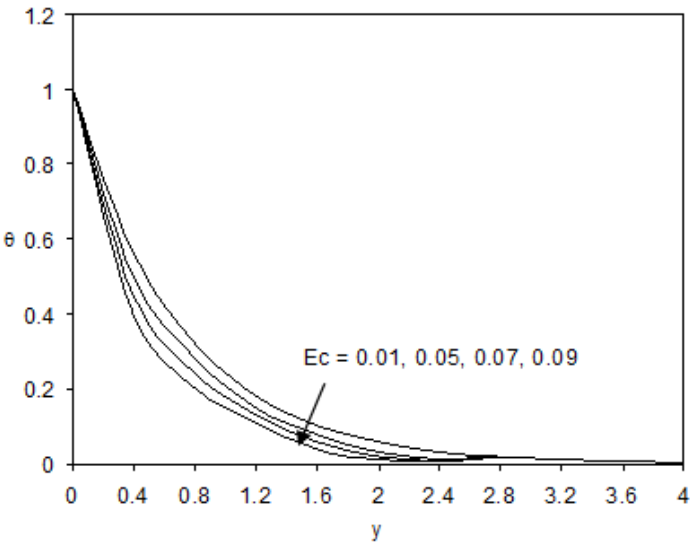

Fig. 8: V ariation of $\theta$ with Ec $\mathrm{G}=10^{3}, \mathrm{M}=2, \mathrm{D}^{-1}=2, \mathrm{~N}=1, \mathrm{~S}_{\mathrm{c}}=1.3, \mathrm{~S}_{\mathrm{r}}=0.5$ $\mathrm{K}=0.5, \alpha=2, \mathrm{t}=0.1$ 

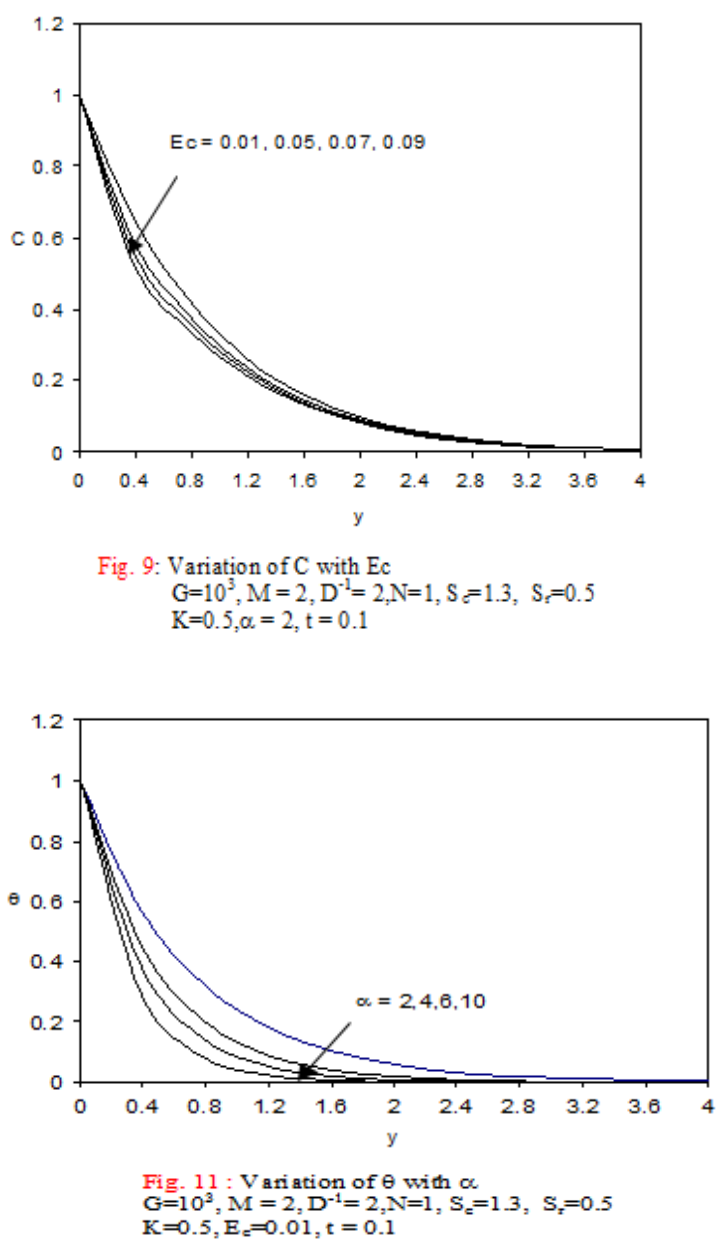

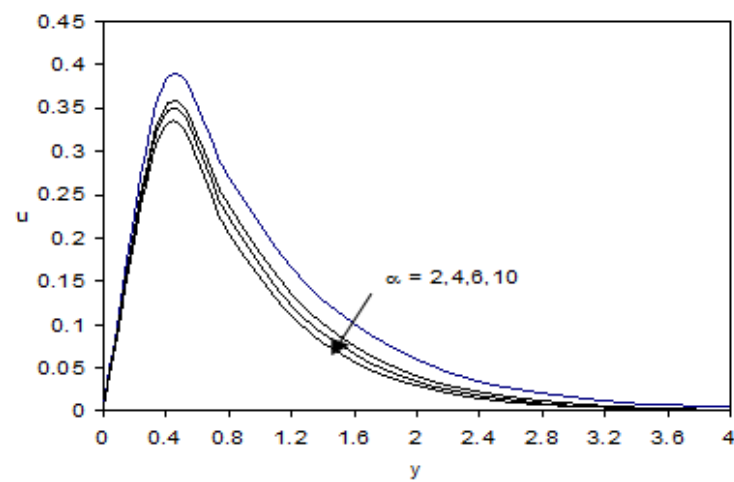

Fig. 10: Variation of $u$ with $\alpha$ $=2, \mathrm{~N}=1, \mathrm{~S}_{\mathrm{s}}=1.3, \mathrm{~S}_{\mathrm{r}}=0.5$ $\mathrm{K}=0.5, \mathrm{E}_{\mathrm{s}}=0.01, \mathrm{t}=0.1$

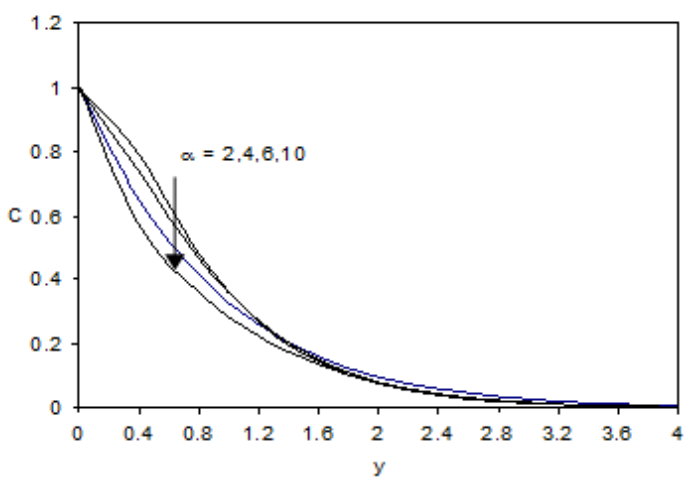

Fig. 12 : V ariarion of $\mathrm{C}$ with $\alpha$ $\mathrm{G}=10^{3}, \mathrm{M}=2, \mathrm{D}^{-1}=2, \mathrm{~N}=1, \mathrm{~S}_{c}=1.3, \mathrm{~S}_{z}=0.5$

The effect of thermo - diffusion on $\mathrm{u}$ is shows in fig.1. It is found that higher the value of $|\mathrm{Sr}| \operatorname{larger}|\mathrm{u}|$ in the boundary layer (fig.1). An increase in the Soret parameter leads to a depreciation in the temperature (fig.2).From fig.3 we find that the concentration depreciates with $\mathrm{Sr} \leq 1.5$ and enhances with higher $\mathrm{Sr} \geq 2.5$, while it enhances with $|\mathrm{Sr}|$ in the entire flow region.From fig.4 we find that the velocity experiences depreciation in the degenerating chemical reaction case.

Fig. 5 shows the variation of $\theta$ with chemical reaction parameter K. It can be seen from the profiles that the actual temperature enhances in the degenerating chemical reaction case. An increase in $\mathrm{K}$ leads to a depreciation in $\mathrm{C}$ (fig. 6).

The effect of a dissipation on $\mathrm{u}$ is exhibited in fig.7 .The Eckert number Ec expresses the relationship between the kinetic energy in the flow and the enthalpy. It embodies the conversion of kinetic energy into internal energy by work done against the viscous fluid stresses. The positive Eckert number implies cooling of the plate i.e loss of heat from the plate to the fluid. Hence, greater viscous dissipation heat causes a depreciation in the velocity.Higher the viscous dissipation heat smaller the actual temperature in the boundary layer (fig.8). An increase in Ec leads to a depreciation in C (fig. 9).Fig.10 represents $u$ with heat source parameter $\alpha$. It is found that higher the strength of the heat source smaller the velocity in the boundary layer.

An increase in $\alpha(>0)$ results in a depreciation in $\theta$. Thus the presence of the heat sources in the flow region reduces the temperature in the flow region (fig.11).

From fig. 12 we find that the actual concentration reduces with $\alpha \leq 4$ and enhances with higher $\alpha \geq 6$. 
Effect Of Thermo- Diffusion And Dissipation On Double Diffusive Heat And Mass Transfer Flow Of..

Table - 1

Shear stress $(\tau)$ at $y=0$

\begin{tabular}{|c|c|c|c|c|c|c|}
\hline $\mathbf{G}$ & I & II & III & IV & V & VI \\
\hline $10^{3}$ & 3.60205 & 3.74875 & 4.11637 & 3.01351 & 2.64589 & 2.27826 \\
\hline $3 \times 10^{3}$ & 10.79402 & 11.23412 & 12.337 & 9.02839 & 7.92553 & 6.82266 \\
\hline$-10^{3}$ & -3.58992 & -3.73662 & -4.10424 & -3.00138 & -2.63376 & -2.26613 \\
\hline$-3 \times 10^{3}$ & -10.78189 & -11.222 & -12.3249 & -9.01626 & -7.91339 & -6.81053 \\
\hline $\mathrm{Sr}$ & 0.5 & 1.5 & 2.5 & -0.5 & -1.5 & -2.5 \\
\hline
\end{tabular}

T able - 2

Shear stress $(\tau)$ at $y=0$

\begin{tabular}{|c|c|c|c|c|c|c|c|}
\hline $\mathbf{G}$ & I & II & III & IV & V & VI & VII \\
\hline $10^{3}$ & 3.38113 & 3.28536 & 3.21638 & 3.39326 & 3.41752 & 3.3084 & 3.26145 \\
\hline $3 \times 10^{3}$ & 10.1313 & 9.84395 & 9.63702 & 10.14339 & 10.1677 & 9.91306 & 9.77221 \\
\hline$-10^{3}$ & -3.369 & -3.27323 & -3.2043 & -3.35687 & -3.33261 & -3.29626 & -3.2493 \\
\hline$-3 \times 10^{3}$ & -10.1191 & -9.62489 & -9.6249 & -10.107 & -10.0827 & -9.90092 & -9.7601 \\
\hline $\mathrm{K}_{\mathrm{r}}$ & 0.5 & 1 & 1.5 & 0.5 & 0.5 & 0.5 & 0.5 \\
\hline $\mathrm{E}_{\mathrm{c}}$ & 0.01 & 0.01 & 0.01 & 0.03 & 0.07 & 0.01 & 0.01 \\
\hline$\alpha$ & 2 & 2 & 2 & 2 & 2 & 4 & 6 \\
\hline
\end{tabular}

The skin friction $(\tau)$ at $\mathrm{y}=0$ is depicted in tables $1-2$ for different values of $\mathrm{S}_{\mathrm{r}}, \mathrm{K}_{\mathrm{r}}, \mathrm{E}_{\mathrm{c}}, \alpha$. The magnitude of $\tau$ enhances with increase in $S_{r}>0$ and reduces with $\left|S_{r}\right|(<0)$ (table 1).

An increase in $\mathrm{K}$ or $\alpha$ reduces $|\tau|$. Also $|\tau|$ enhances with increase in Eckert number $\mathrm{E}_{\mathrm{c}}$. (table 2).

Table - 3

Sherwood Number (Sh) at $\mathrm{y}=0$

\begin{tabular}{|c|c|c|c|c|c|c|}
\hline $\mathbf{G}$ & I & II & III & IV & V & VI \\
\hline $10^{3}$ & 0.05231 & -25.1257 & -66.9701 & -5.26524 & 25.6062 & 70.5738 \\
\hline $3 \times 10^{3}$ & -0.35859 & -36.0984 & -89.1292 & 10.91665 & 113.387 & 437.496 \\
\hline$-10^{3}$ & 0.36335 & -13.6595 & -48.7106 & 3.1449 & 16.6926 & 54.8669 \\
\hline$-3 \times 10^{3}$ & 0.57455 & -67.0056 & -96.1482 & 4.55561 & 86.6457 & 390.375 \\
\hline $\mathrm{S}_{\mathrm{r}}$ & 0.5 & 1.5 & 2.5 & -0.5 & -1.5 & -2.5 \\
\hline
\end{tabular}

Table - 4

Sherwood Number (Sh) at $\mathrm{y}=0$

\begin{tabular}{|c|c|c|c|c|c|c|c|}
\hline $\mathbf{G}$ & I & II & III & IV & V & VI & VII \\
\hline $10^{3}$ & -6.34634 & -3.22176 & -2.1806 & -6.8387 & -7.82343 & 6.03599 & 4.63377 \\
\hline $3 \times 10^{3}$ & -12.9887 & -6.52919 & -2.8098 & -17.42 & -26.2826 & -11.1216 & 2.96094 \\
\hline$-10^{3}$ & -1.6734 & -2.03916 & 2.68332 & -2.16577 & -3.1505 & 2.70971 & 3.48244 \\
\hline$-3 \times 10^{3}$ & 1.03007 & 5.78186 & 6.18186 & -3.40122 & -12.2638 & -21.1004 & -4.49303 \\
\hline $\mathrm{K}_{\mathrm{r}}$ & 0.5 & 1 & 1.5 & 0.5 & 0.5 & 0.5 & 0.5 \\
\hline $\mathrm{E}_{\mathrm{c}}$ & 0.01 & 0.01 & 0.01 & 0.03 & 0.07 & 0.01 & 0.01 \\
\hline$\alpha$ & 2 & 2 & 2 & 2 & 2 & 4 & 6 \\
\hline
\end{tabular}

The rate of mass transfer (Sherwood number) at the plate $y=0$ is shown in tables $3 \& 4$ for different parametric values. From table 3 we find that the rate of mass transfer enhances with increase in Soret parameter $\left|\mathrm{S}_{\mathrm{r}}\right|$ for all $\mathrm{G}$. An increase in $\mathrm{K}$ or $\alpha$ reduces $|\mathrm{Sh}|$ for $\mathrm{G}>0$ and for $\mathrm{G}<0,|\mathrm{Sh}|$ experiences an enhancement at $\mathrm{y}=0$ (table 4 ). 


\section{Conclusions}

$>$ Higher the value of $|\mathrm{Sr}|$ larger $|\mathrm{u}|$ in the boundary layer.

$>$ An increase in the Soret parameter leads to a depreciation in the temperature. the concentration depreciates with $\mathrm{Sr} \leq 1.5$ and enhances with higher $\mathrm{Sr} \geq 2.5$, while it enhances with $|\mathrm{Sr}|$ in the entire flow region.

$>$ The kinetic energy in the flow and the enthalpy. It embodies the conversion of kinetic energy into internal energy by work done against the viscous fluid stresses.

> Higher the viscous dissipation heat smaller the actual temperature in the boundary layer.

$>\mathrm{An}$ increase in Ec leads to a depreciation in C.

$>$ The magnitude of $\tau$ enhances with increase in $\operatorname{Sr}>0$ and reduces with $|\mathrm{Sr}|(<0)$.

$>|\tau|$ enhances with increase in Eckert number Ec.

The rate of mass transfer enhances with increase in Soret parameter $|\mathrm{Sr}|$ for all G.

\section{References}

[1]. Abdus-Sattar, M. D. and Hamid Kalim, M. D. :J. Math. Phys. Sci. 30 PP.25 - 37 (1996).

[2]. Cookey, C. I.; Ogulu, A. and Omubo-Pepple, V. M. Int. J. Heat Mass Transfer 46 PP.2305 -2311(2003).

[3]. Ganesan, P. and Loganathan, P. : Radiation and mass transfer effects on flow of an incompressible viscous fluidNpast a moving vertical cylinder, Int. J. of Heat and Mass Transfer 45 PP.4281- 4288(2002).

[4]. GireeshKumar.J\&Ramakrishna.S, Effect of chemical reaction and mass transfer on radiation and MHD free convection flow of Kuvshinski fluid through porous medium, J.Pure\& Appl.Phys.,Vol.22(3), pp.431-441(2010).

[5]. Hassanien, I. A. and Obied Allah, M. H. :Int. Comm. Heat mass transfer 29 (4) PP.567-575(2002).

[6]. Kim, Y. J. :Int. J. Eng. Sci. 38 PP.833- 845(2000).

[7]. Makinde, O. D. :Int. Comm Heat Mass Transfer 32PP.1411-1419(2005).

[8]. Mansour, M. A. : Astrophys. SpaceSci. 166 PP.269 - 275(1990).

[9]. Mbeledogu, I. U.; Amakiri, A. R. C. and Ogulu, A. :Int. J. of Heat and Mass Transfer 50 PP.1668-1674(2007).

[10]. Mohamed, R.A. :Applied Mathematical Sciences, Vol.3, No. 13 PP.629-651(2009).

[11]. Muthucumaraswamy, R. and Ganesan, P. :J. Appl. Mech. Tech. Phys. 42 PP. $665-671(2001)$.

[12]. Raptis, A. ; Perdikis, C. and Leontitsis, A. : Heat Mass Transfer 39 PP.771 - 773(2003).

[13]. Raptis, A. and Perdikis, C. :Appl. Mech. Eng. 4 (4) PP.817 - 821(1999).

[14]. Raptis, A. and Perdikis, C. :Int. J. of Non-Linear Mechanics 41 PP.527-529(2006).

[15]. Takhar, H. S.; Gorla, R. S. R. and Soundalgekar, V. M. :Int. J. Numer. Methods Heat Fluid Flow 6 (2) PP.77 - 83(1996).

[16]. Vidyasagar B, Raju M.C, Varma S.V.K (2015) Unsteady MHD Free Convection Flow of a Viscous Dissipative Kuvshinski Fluid Past an Infinite Vertical Porous Plate in the Presence of Radiation, Thermal Diffusion and Chemical Effects. J ApplComputat Math 4:255. doi:10.4172/2168-9679.1000255.

[17]. B. Vidyasagar, M. C. Raju, S.V. K.Varma, S.V. Ramana, Unsteady MHD free convection boundary layer flow of radiation absorbing Kuvshinski fluid through porous medium, Review of Advances in Physics Theories and Applications, 2014, 1(3): 4862..4172/2168-9679.1000255.

[18]. S. Harinath Reddy, M. C. Raju, E. Keshava Reddy, Unsteady MHD free convection flow of a Kuvshinski fluid past a vertical porous plate in the presence of chemical reaction and heat source/sink, International Journal of Engineering Research in Africa Vol. 14 (2015) pp. 13-27, doi:10.4028/www.scientific.net/JERA.14.13.

[19]. V. Ravikumar, M. C. Raju, G. S. S. Raju., MHD three dimensional Couette flow past a porous plate with heat transfer, IOSR Jour. Maths., Vol. 1, no.3, pp. 3-9, 2012.

[20]. M. Umamaheswar, S. V. K. Varma, M. C. Raju, Numerical study of Magneto-Convective and radiation absorption fluid flow past an exponentially accelerated vertical porous plate with variable temperature and concentration in the presence of Soret and Dufour effects, IOSR Journal of Mathematics, Volume 12, Issue 2 Ver. I (Mar. -Apr.2016), PP 109-120. DOI: 10.9790/5728-1221109120.

[21]. B. M. Rao, G. V. Reddy, M. C. Raju, S. V. K. Varma, MHD transient free convection and chemically reactive flow past a porous vertical plate with radiation and temperature gradient dependent heat source in slip flow regime, IOSR Journal of Applied Physics, Vol. 3(6), 2013, 22-32.

[22]. V. Ravikumar, M. C. Raju, G. S. S. Raju, Magnetic field and radiation effects on a double diffusive free convective flow bounded by two infinite impermeable plates in the presence of chemical reaction, IJSER Volume 4, Issue 7, pp. 1915-1923,2013.

[23]. V. Ravikumar, M. C. Raju, G. S. S. Raju, A. J. Chamkha, "MHD double diffusive and chemically reactive flow through porous medium bounded by two vertical plates", International Journal of Energy \& Technology, 5 (4), 2013, pp. 1-8.

[24]. B. M. Rao, M. C. Raju, G.V. Reddy, S.V.K. Varma, Unsteady MHD Free Convective Double Diffusive and Dissipative ViscoElastic Fluid Flow in Porous Medium with Suction, International Journal of Advances in Science and Technology,Vol.7, No.2, 2013.

[25]. L. Rama Mohan Reddy, M. C. Raju, G. S. S. Raju, Natural convection boundary layer flow of a double diffusive and rotating fluid past a vertical porous plate. Int. J. Appl. Comput. Math. (2016). DOI 10.1007/s40819-n-0174-7.

[26]. P. Chandrareddy, M. C. Raju, G. S. S. Raju, Magnetohydrodynamic Convective Double Diffusive Laminar Boundary Layer Flow Past an Accelerated Vertical Plate, International Journal of Engineering Research in Africa, Vol. 20 (2016), 80-92, doi:10.4028/www.scientific.net/JERA.20.80.

[27]. M. Umamaheswar, S. V. K. Varma, M. C. Raju, A.J. Chamkha., Unsteady MHD Free Convective Double Diffusive Visco-Elastic Fluid Flow Past an Inclined Permeable Plate in the presence of viscous dissipation and heat absorption, Special topics and Reviews in Porous media: An international Journal., Vol.6 (4). 2016, pp. 333-342.

[28]. M. C. Raju, and S.V. K.Varma, Soret effects due to natural convection in a non-Newtonian fluid flow in porous medium with heat and mass transfer, Journal of Naval architecture and Marine Engineering, Vol. 11 (2), 2014, pp. 147-156.DOI: 10.3329/jname.v6i1.2654.

[29]. B. Mamtha, M. C. Raju, S.V.K.Varma, Thermal diffusion effect on MHD mixed convection unsteady flow of a micro polar fluid past a semi-infinite vertical porous plate with radiation and mass transfer, International Journal of Engineering Research in Africa, Vol. 13 (2015) pp 21-37.

[30]. Chandra, R.P, Raju, M.C, Raju, G.S.S., (2015). Thermal and Solutal Buoyancy Effect on MHD Boundary Layer Flow of a ViscoElastic Fluid Past a Porous Plate with Varying Suction and Heat Source in the Presence of Thermal Diffusion. J ApplComputat Math 4: 249. doi:10.4172/2168-9679.1000249. 